\title{
Laboratory Evaluation of Phenotypic Detection Methods of Methicillin-Resistant Staphylococcus Aureus
}

\author{
Arunava Kali, Selvaraj Stephen, Sivaraman Umadevi
}

\begin{abstract}
Although conventional antibiotic susceptibility tests are most commonly performed for methicillin-resistant Staphylococcus aureus (MRSA), the results of these phenotypic tests are dependent on the standardization of the culture conditions. The aim of the study was to evaluate the conventional phenotypic screening tests in comparison to the mecA gene polymerase chain reaction (PCR). One hundred and two clinical isolates of MRSA identified by the oxacillin disk diffusion were subjected to PCR for the mecA gene and by the cefoxitin disk diffusion test and culture on oxacillin screen agar, mannitol salt agar, and methicillin-resistant Staphylococcus aureus Agar (MeReSA) selective medium, for MRSA. Although all 102 isolates were resistant in oxacillin and cefoxitin disk diffusion, $92(90.1 \%)$ isolates were positive for the $m e c A$ gene. The sensitivities of the mannitol salt agar, MeReSA agar, and oxacillin screen agar were $89.13,97.82$, and $98.91 \%$, respectively. The oxacillin screen agar may be recommended for confirming methicillin resistance in the disk diffusion test in resource-poor settings, where molecular methods are not available.
\end{abstract}

(Biomed J 2014;37:411-414)

Key words: Cefoxitin disk diffusion, methicillin-resistant Staphylococcus aureus, mecA gene, oxacillin disk diffusion, oxacillin screen agar, polymerase chain reaction

A ntibiotic therapy for patient care as well as hospital infection control measures essentially rely on the laboratory for accurate antimicrobial sensitivity results. Failure to report methicillin resistance in Staphylococcus aureus (S. aureus) may result in treatment failure, poor prognosis, increased cost of treatment, and dissemination of multi-drug resistant strains. Although molecular methods are considered to be the gold standard, ${ }^{[1]}$ conventional antibiotic susceptibility tests are the mainstream of MRSA laboratory diagnosis in resource-limited settings. Phenotypic tests have their own disadvantages, as these are affected by culture conditions. ${ }^{[1-3]}$ Various selective media containing methicillin, oxacillin, and cefoxitin are available commercially and commonly used for MRSA surveillance cultures. Currently, the Clinical and Laboratory Standards Institute (CLSI) recommends cefoxitin as the sole marker of mecA gene-mediated oxacillin resistance and use of oxacillin disk diffusion has been discontinued. ${ }^{[3]}$ This study has been carried out to evaluate different conventional phenotypic screening tests for MRSA in comparison to PCR detection of the mecA gene.

\section{METHODS}

This cross-sectional study was carried out in the Department of Microbiology, in a tertiary care hospital in South India after Institutional Ethical Committee clearance. All clinical samples were processed in the laboratory as per the standard guidelines. S. aureus isolates were identified by gram stain, colony morphology on blood agar, and MacConkey agar, catalase, and coagulase and deoxyribonuclease (DNase) test. The disk diffusion test (DDT) using a $1 \mu \mathrm{g}$ oxacillin disk was employed as the initial screening test. Consecutive isolates from the same patient were excluded. Oxacillin-resistant $S$. aureus isolates were subjected to cefoxitin $(30 \mu \mathrm{g})$ DDT, culture on oxacillin screen agar (containing $6 \mu \mathrm{g} / \mathrm{ml}$ oxacillin and $4 \% \mathrm{NaCl}$ ), mannitol salt agar with cefoxitin and MeReSA agar (HiMedia, Mumbai, India), and PCR for the mecA gene.

The phenol-chloroform extraction method was used for DNA extraction. Each PCR reaction mixture $(25 \mu \mathrm{l})$ contained, $2 \mu \mathrm{l}$ of 1 PCR buffer, $2.0 \mathrm{mM}$ of each of the primers

From the Department of Microbiology, Mahatma Gandhi Medical College and Research Institute, Pondicherry, India Received: Sep. 12, 2013; Accepted: Dec. 30, 2013

Correspondence to: Dr. Arunava Kali, Department of Microbiology, Mahatma Gandhi Medical College and Research Institute, India. Pondicherry - 607 402, India. Tel: 91-9868331408; Fax: 91-011-29942265; E-mail: ak.arunava@ gmail.com

DOI: $10.4103 / 2319-4170.132907$ 
$(1 \mu \mathrm{l}), 15 \mathrm{mM}$ of each deoxynucleotide triphosphate $(1 \mu \mathrm{l})$, Taq DNA polymerase $(1 \mu \mathrm{l}), 14 \mu \mathrm{l}$ of molecular grade water, and a DNA template $(2 \mu \mathrm{l})$. The forward and reverse primers were 5'- AAAATCGATGGTAAAGGTTGGC-3' and 5'-AGTTCTGCAGTACCGGATTTGC-3', respectively. The amplification protocol included five minutes of initial denaturation at $95^{\circ} \mathrm{C}$, followed by 35 cycles of denaturation at $95^{\circ} \mathrm{C}$ for 60 seconds, annealing at $54^{\circ} \mathrm{C}$ for 60 seconds, and extension for 60 seconds at $72^{\circ} \mathrm{C}$, with a final extension at $72^{\circ} \mathrm{C}$ for 10 minutes. Following PCR, the reaction mixtures were analyzed for 533 base pair amplified products by electrophoresis on a $1 \%$ agarose gel containing ethidium bromide $(0.2 \mathrm{mg} / \mathrm{ml})$, in the presence of an appropriate DNA molecular weight marker.

S. aureus ATCC 25923 and ATCC 43300 were used as controls for the antibiotic susceptibility test and PCR. The viability of the test isolates was maintained by a periodic subculture in a semisolid nutrient agar. All data were entered in a Microsoft Excel 2007 spreadsheet and statistical analysis was done. For categorical variables, the proportions are represented as percentages. The sensitivity, specificity, and positive and negative predictive values were calculated. True positive (TP) is defined as positive results in the new test under evaluation, which are also positive by the gold standard test. ${ }^{[4]}$ Similarly, true negative $(\mathrm{TN})$ indicates negative results in the new test as well as by the gold standard test. False positive (FP) and false negative (FN) are positive and negative results, respectively, detected by the new test, which differ from the gold standard test results. Sensitivity is the ability of a test to correctly classify a test result as 'positive'. However, the ability of a test to correctly classify a test result as 'negative' is called specificity. A positive predictive value is the percentage of true positive results out of the total positives (both true and false positives) obtained by the test under evaluation. Likewise, a negative predictive value is the percentage of true negative results out of the total negatives (both true and false negatives) obtained by the new test. The following statistical formulas were used ${ }^{[4]}$

Sensitivity $=\mathrm{TP} /(\mathrm{TP}+\mathrm{FN})$

Specificity $=\mathrm{TN} /(\mathrm{FP}+\mathrm{TN})$

Positive predictive value $=\mathrm{TP} /(\mathrm{TP}+\mathrm{FP})$

Negative predictive value $=\mathrm{TN} /(\mathrm{TN}+\mathrm{FN})$

Accuracy $=(\mathrm{TP}+\mathrm{TN}) /(\mathrm{TP}+\mathrm{TN}+\mathrm{FP}+\mathrm{FN})$.

\section{RESULTS}

One hundred and two non-duplicate oxacillin-resistant S. aureus isolates from various clinical samples identified by oxacillin disk diffusion were included in this study. The comparison of various phenotypic methods with PCR for the mecA gene is represented in Table 1.

All 102 isolates were resistant in oxacillin and cefoxitin DDT. Among these isolates, 89 (87.2\%), 97 (95.1\%), and
$92(90.1 \%)$ isolates showed growth in the mannitol salt agar with cefoxitin, MeReSA agar, and OSA, respectively. The PCR assay for the mecA gene detected $92(90.1 \%)$ mecA positive and 10 (9.8\%) mecA negative isolates. Out of the $92 \mathrm{mec} A$ PCR-positive isolates, 82, 90, and 91 strains were correctly detected as MRSA using the mannitol salt agar, MeReSA agar, and OSA, respectively. The sensitivity of MSA, MeReSA, and OSA selective media was $89.13,97.82$, and $98.91 \%$, respectively. Among the $10 \mathrm{mecA}$ negative isolates, seven were incorrectly identified as oxacillin-resistant by MSA and MeReSA. The low specificity of MSA and MeReSA may be attributed to these high false positive results. However, MeReSA was superior with lower false negative results $(n=2)$ in comparison to MSA $(n=10)$. OSA displayed only one false positive and one false negative result. Consequently, OSA had $98.91 \%$ sensitivity and $90 \%$ specificity. With respect to PCR as the gold standard test, the accuracy of MSA, MeReSA, and OSA was $83.33,91.11$, and $98.04 \%$, respectively.

\section{DISCUSSION}

Rapid and accurate detection of MRSA has a decisive role in hospital-infection control. MRSA can be detected either by demonstration of the mecA gene and its product PBP2a or by demonstration of phenotypic characters conferred by the mecA gene, namely, resistance to beta-lactam antibiotics in terms of increased minimum inhibitory concentration (MIC) or decreased zone of inhibition for oxacillin and cefoxitin. ${ }^{[1]}$ Unlike the phenotypic tests, assays like PCR for the mecA gene, are rapid, accurate, commercially available, and thus, considered the gold standard.$^{[1]}$ However, cost constraints and greater technical and infrastructural requirements curtail their widespread use, especially in resource-poor settings. On the other hand, results of the phenotypic tests are dependent on the standardization of culture conditions like temperature, incubation period, salt concentration, inoculum size, and $\mathrm{pH}$ of the medium. ${ }^{[1-3]}$ The antibiotic susceptibility testing for MRSA is further complicated by the presence of heteroresistance and induc-

Table 1: Comparison of mannitol salt agar, MeReSA agar, and oxacillin screen agar for MRSA

\begin{tabular}{lccc}
\hline & MSA $^{*}$ & MeReSA $^{\dagger}$ & OSA $^{\ddagger}$ \\
\hline True positive & 82 & 90 & 91 \\
False positive & 7 & 7 & 1 \\
True negative & 3 & 3 & 9 \\
False negative & 10 & 2 & 1 \\
Sensitivity (\%) & 89.13 & 97.82 & 98.91 \\
Specificity (\%) & 30 & 30 & 90 \\
Positive predictive value (\%) & 92.13 & 92.78 & 98.91 \\
Negative predictive value (\%) & 23.07 & 60 & 90
\end{tabular}

Abbreviations: *MSA: Mannitol salt agar with cefoxitin; ${ }^{\dagger}$ MeReSA: Commercial chromogenic selective media for MRSA; ${ }^{\ddagger}$ OSA: Oxacillin screen agar 
ible resistance seen among the different isolates. ${ }^{[1,5]}$ These isolates are frequently misdiagnosed as methicillin-sensitive S. aureus (MSSA). Methicillin resistance is principally mediated by the mecA gene, which codes for an altered penicillin binding protein (PBP2a). However, isolates with alterations to the existing PBPs, known as moderately resistant $S$. aureus' (MODSA) and penicillinase hyper-producer strains, referred to as 'borderline oxacillin-resistant S. aureus' (BORSA), were also described. ${ }^{[1,2]}$ MRSA with the mecC gene, a novel homolog of mecA, has been reported in the human and bovine population in UK and Denmark. ${ }^{[6]}$ These isolates are $m e c A$ negative in PCR, but resistant in oxacillin DDT. ${ }^{[7]}$ Hence, there have been substantial changes in the CLSI disk diffusion interpretation criteria for MRSA in recent years. Previously cefoxitin DDT or MIC was recommended for isolates with oxacillin-intermediate zones. ${ }^{[3]}$ However, the CLSI 2013 guideline no longer recommends the use of oxacillin DDT, as it may not effectively reflect the mecA-mediated resistance mechanism. ${ }^{[3]}$

In this present study, we compared three selective media for MRSA, namely, MSA, MeReSA, and OSA, with a genotypic method. PCR for mecA is the universally accepted gold standard detection method for MRSA. However, its sensitivity, specificity, and accuracy depend on the selection of primers, quality of reagents, and the amplification protocol used. In our study, we used a primer set that yielded 533 base pair amplified products, as described by Murakami et al. ${ }^{[8]}$ Different primer sets and amplification protocols have been used in various studies. ${ }^{[2,9]}$ We detected that 92 isolates were $m e c A$ positive and the remaining 10 were $m e c A$ negative. Compared to the previous studies, a higher proportion of oxacillin-resistant (oxacillin disk diffusion test) strains were found to be mecA PCR negative. ${ }^{[7-9]}$ However, our results are in accordance with a similar study from South India where out of 75 oxacillin-resistant strains only 62 were mecA positive and 13 were $m e c A$ negative. ${ }^{[2]}$ This inconsistency in results of various studies may be attributed to the difference in primers and amplification protocols as well as regional variation of the MRSA strains.

We found OSA to be the most suitable alternative for molecular methods, as it had $98.91 \%$ sensitivity. Among all three selective media used, mannitol salt agar showed the lowest sensitivity (89.13\%). Several strains of MRSA might fail to grow on MSA due to its high salt concentration. ${ }^{[1]}$ Out of 13 strains that failed to grow on MSA after 24 hours, 10 strains showed scanty growth after prolonged incubation for 48-72 hours. Other authors also reported similar findings. ${ }^{[2,10]}$ Cefoxitin-supplemented MSA were reported to have better sensitivity and specificity than MSA and MSA with oxacillin. ${ }^{[10]}$ Yamazumi et al., reported 96.9, 98, 98, and 99\% sensitivities and 100, 98, 100, and 99\% specificities for MRSA-Screen agar, OSA, Vitek, and the microdilution test, respectively. ${ }^{[11]}$ Cefoxitin has been reported to be superior to oxacillin by authors on several occasions. ${ }^{[7,9,12,13]}$ Unlike the oxacillin disk, the zones of inhibition of the cefoxitin disk are distinct, making it easier to interpret. ${ }^{[7]}$ Broekema et al., found that the sensitivity and specificity of cefoxitin DDT was 97.3 and $100 \%$ compared to the oxacillin disk. ${ }^{[7]}$ In another study, cefoxitin DDT had $100 \%$ sensitivity in contrast to the 87.5 and $96.8 \%$ sensitivities of oxacillin DDT and OSA, respectively ${ }^{\left[{ }^{[9]}\right.}$ As per the current CLSI guideline cefoxitin DDT and agar dilution specifically identified $m e c A$-mediated resistance. However, we found no difference in the results of cefoxitin and oxacillin DDT. All 102 isolates were resistant to both cefoxitin and oxacillin, and in both tests, $10 \mathrm{mecA}$ negative isolates were falsely detected as resistant. These 10 false positive results could be attributed to the presence of alternate resistance mechanisms (MODSA and BORSA) in these isolates. However, there were no fundamental differences in the clinical course and treatment outcome of these cases. In this study, a substantial proportion of the isolates were $m e c A$ negative, which could express alternate resistance mechanisms. Although non-mecA-mediated methicillin resistance in S. aureus has been reported to be $1.3 \%,{ }^{[7]}$ there are infrequent reports from the Indian setup. However, further large-scale studies are essential to confirm it.

The limitation of the study was the small sample size. As we had selectively included MRSA strains by choosing only oxacillin-resistant isolates, the number of $m e c A$ negative isolates was very small. Hence, the specificity might not have an implication.

In conclusion, we found that a significant proportion of MRSA isolates detected by oxacillin and cefoxitin DDT was negative for the mecA gene. Although the clinical significance was unknown, these isolates might possess alternate resistance mechanisms like hyperproduction of penicillinase or alteration of the existing PBP.

We report the oxacillin screen agar as the best alternative for PCR and recommend using the oxacillin screen agar for confirming methicillin resistance in resource-limited settings.

\section{REFERENCES}

1. Brown DF, Edwards DI, Hawkey PM, Morrison D, Ridgway GL, Towner KJ, et al. Guidelines for the laboratory diagnosis and susceptibility testing of methicillin-resistant Staphylococcus aureus (MRSA). J Antimicrob Chemother 2005;56:1000-18.

2. Pillai M, Latha R, Sarkar G. Detection of methicillin resistance in staphylococcus aureus by polymerase chain reaction and conventional methods: A comparative study. J Lab Physicians 2012;4:83-8.

3. Clinical and Laboratory Standards Institute. Performance standards for antimicrobial susceptibility testing: $23^{\text {rd }}$ informational supplement. CLSI document M100-S23. Wayne, Pennsylvania: Clinical and Laboratory Standards Institute; 2013.

4. Parikh R, Mathai A, Parikh S, Sekhar GC, Thomas R. Understanding 
and using sensitivity, specificity and predictive values. Indian $\mathrm{J}$ Ophthalmol 2008;56:45-50.

5. Chambers HF. Methicillin resistance in Staphylococci: Molecular and biochemical basis and clinical implications. Clin Microbiol Rev 1997; 10:781-91.

6. Garcia-Alvarez L, Holden MT, Lindsay H, Webb CR, Brown DF, Curran MD, et al. Meticillin-resistant Staphylococcus aureus with a novel mecA homologue in human and bovine populations in the UK and Denmark: A descriptive study. Lancet Infect Dis 2011;11:595-603.

7. Broekema NM, Van TT, Monson TA, Marshall SA, Warshauer DM. Comparison of cefoxitin and oxacillin disk diffusion methods for detection of mecA-mediated resistance in Staphylococcus aureus in a large-scale study. J Clin Microbiol 2009;47:217-9.

8. Murakami K, Minamide W, Wada K, Nakamura E, Teraoka H, Watanabe S. Identification of methicillin-resistant strains of staphylococci by polymerase chain reaction. J Clin Microbiol 1991;29:2240-4.

9. Anand KB, Agrawal P, Kumar S, Kapila K. Comparison of cefoxitin disk diffusion test, oxacillin screen agar, and PCR for mecA gene for detection of MRSA. Indian J Med Microbiol 2009;27:27-9.

10. Stoakes L, Reyes R, Daniel J, Lennox G, John MA, Lannigan R, et al. Prospective comparison of a new chromogenic medium, MRSASelect, to CHROMagar MRSA and mannitol-salt medium supplemented with oxacillin or cefoxitin for detection of methicillin-resistant Staphylococcus aureus. J Clin Microbiol 2006;44:637-9.

11. Yamazumi T, Marshall SA, Wilke WW, Diekema DJ, Pfaller MA, Jones RN. Comparison of the Vitek Gram-Positive Susceptibility 106 card and the MRSA-screen latex agglutination test for determining oxacillin resistance in clinical bloodstream isolates of Staphylococcus aureus. J Clin Microbiol 2001;39:53-6.

12. Arya SC, Mehta LK, Mathai G, Agarwal BK, Agarwal S. Evaluation of oxacillin and cefoxitin disk diffusion test for routine detection of methicillin resistant Staphylococcus aureus. Saudi Med J 2005;26:2009-10.

13. Sharp SE, Warren JA, Thomson RB Jr. Cefoxitin disk diffusion screen for confirmation of oxacillin-resistant Staphylococcus aureus isolates and utility in the clinical laboratory. Diagn Microbiol Infect Dis 2005;51:69-71. 